\title{
Mitomicina C e "Excimer laser"
}

\author{
Mitomycin Cand Excimer Laser
}

\author{
Anelise Dutra Wallau ${ }^{1}$ \\ Maria Cristina Ventura Leoratti ${ }^{2}$ \\ Mauro Campos ${ }^{3}$
}

Trabalho realizado na Universidade Federal de São Paulo (UNIFESP)

${ }^{1}$ Oftalmologista, Pós-Graduanda da Universidade Federal de São Paulo (UNIFESP). São Paulo (SP).

2 Oftalmologista, Colaboradora do Setor de Cirurgia Refrativa da UNIFESP. São Paulo (SP).

${ }_{3}^{3}$ Professor Livre Docente, Chefe do Setor de Cirurgia Refrativa do Departamento de Oftalmologia da UNIFESP. São Paulo (SP).

Endereço para correspondência: Anelise Dutra Wallau - Rua Borges Lagoa, 980/32 - São Paulo (SP) CEP 04038-002

E-mail: anewallau@hotmail.com

Recebido para publicação em 24.05.2005

Versão revisada em 23.06.2005

Aprovação em 31.10.2005

\section{RESUMO}

A mitomicina C é um antimetabólito que atua em nível celular bloqueando a replicação de DNA e RNA e inibindo a síntese protéica. Utilizada em diversas áreas da oftalmologia, recentemente vem sendo empregada como moduladora da resposta cicatricial corneana em cirurgias ópticas/refrativas por "excimer laser". A aplicação única de mitomicina $\mathrm{C}$ associada à cirurgia fotoablativa de superfície corneana tem se mostrado opção segura e eficiente para fins terapêuticos em olhos com opacidade corneana préexistente e/ou profiláticos em olhos com alto risco de desenvolvimento de opacificação corneana pós-operatória. $\mathrm{O}$ uso da droga em cirurgia fotoablativa deve ser cauteloso até que seguimento de longo prazo avalie sua inocuidade tardia. $\mathrm{O}$ presente texto faz revisão dos principais estudos sobre modulação da resposta cicatricial corneana com uso de mitomicina $\mathrm{C}$ em cirurgias ópticas/refrativas de superfície.

Descritores: Córnea; Cicatrização de feridas; Mitomicina/uso terapêutico; Erros de refração/cirurgia; Ceratectomia fotorrefrativa por excimer laser/métodos

\section{INTRODUÇ̃̃̃O}

A mitomicina C (MMC) é um antimetabólito alquilante derivado do Streptomyces caespitosus ${ }^{(1)}$. Atua bloqueando a replicação de DNA e RNA e inibindo a síntese protéica, sem ciclo celular específico de ação ${ }^{(1)}$. Células em rápida atividade proliferativa são mais suscetíveis à ação da droga. Inicialmente usada como agente quimioterápico sistêmico, a MMC é atualmente utilizada na oftalmologia em cirurgias de glaucoma ${ }^{(2)}$ e $_{\text {pterígio }}{ }^{(3)}$, para tratamento de neoplasias de conjuntiva e córnea ${ }^{(4)}$, em penfigóide ocular cicatricial $^{(5)}$ e recentemente em cirurgias fotoablativas de superfície como moduladora da resposta cicatricial corneana ${ }^{(6-26)}$.

$\mathrm{Na}$ córnea, a mitomicina $\mathrm{C}$ age induzindo apoptose de ceratócitos no estroma anterior, inibindo a ativação, proliferação e migração de ceratócitos e sua transformação fenotípica em fibroblastos e miofibroblastos, evitando a formação de colágeno desorganizado e "haze"(6-10). A ação da droga é dependente da concentração e do tempo de exposição da mesma à superfície corneana; o equilíbrio ideal entre estes dois fatores resultaria em maior efeito antiproliferativo/citostático (inibição da ativação e proliferação de ceratócitos) e



Em cirurgia óptica/refrativa, a mitomicina $\mathrm{C}$ per-operatória vem sendo utilizada como terapia adjunta no tratamento de "haze" e fibrose subepitelial corneana associada ou não à ceratectomia fototerapêutica (PTK), em ceratectomia fotorrefrativa (PRK) após complicações com "flap" de "laser-assisted in situ keratomileusis" (LASIK), em PRK pós-ceratotomia radial, PRK póstransplante de córnea e mais recentemente como profilaxia à formação de "haze" em PRK e olhos suscetíveis sem cirurgia refrativa prévia. 
Revisaremos a seguir os principais estudos publicados sobre modulação da resposta cicatricial corneana com uso de mitomicina $\mathrm{C}$.

\section{Estudos experimentais}

Talamo et al., em 1991 foram os primeiros a sugerir o uso de mitomicina $\mathrm{C}$ como modulador da cicatrização corneana ${ }^{(11)}$. Após ablação corneana de $100 \mu \mathrm{m}$ em coelhos, olhos recebendo mitomicina C $0,05 \%$ como colírio duas vezes ao dia durante 14 dias apresentaram redução significativa na síntese de colágeno subepitelial e fibrose.

O primeiro experimento foi descrito em 1997 por Schipper et al., com aplicação de dose única de mitomicina $\mathrm{C}$ no leito estromal corneano de coelhos ${ }^{(6)}$. Utilizaram mitomicina $0,04 \%$ por cinco minutos após ablação corneana de aproximadamente $80 \mu \mathrm{m}$. Observaram redução significativa na formação de cicatriz corneana e no número de ceratócitos, sem diferença significativa quanto à presença de "haze".

Estudo "in vitro" com ceratócitos humanos em meio de cultura para quantificar ação antiproliferativa e citotóxica da MMC verificou que os efeitos da droga são dependentes da concentração e do tempo de exposição da mesma às células ${ }^{(7)}$. A dose inibitória média (suficiente para inibir a proliferação de $50 \%$ dos ceratócitos) durante exposição de cinco minutos foi de mitomicina $\mathrm{C} 0,0038 \%$. A dose letal média (suficiente para eliminar 50\% dos ceratócitos) foi de MMC 0,028\% após uma hora de exposição( ${ }^{(7)}$.

McDermott et al., avaliaram os efeitos da MMC sobre o endotélio humano em duas diferentes concentrações ${ }^{(29)}$. Quatro pares de córneas humanas foram submetidos à irrigação apenas com solução salina (quatro córneas, controles) ou solução salina acrescida de MMC 0,002\% (quatro córneas) durante três horas. $\mathrm{O}$ experimento foi repetido com dois pares de córneas (casos e controles) e MMC desta vez 0,02\%. Paquimetria corneana óptica foi realizada a cada 15 minutos em todas as córneas. Ao final das três horas de experimento, as córneas foram avaliadas por microscopia eletrônica. Não houve diferença estatística na paquimetria corneana ou na avaliação por microscopia eletrônica entre o grupo irrigado apenas com solução salina e o grupo que recebeu MMC $0,002 \%$. Perfusão corneana com MMC $0,02 \%$ resultou em edema corneano imediato, com importantes alterações estruturais à microscopia eletrônica. Os autores ponderam que o endotélio corneano humano é relativamente amitótico, porém mesmo células amitóticas necessitam reparo periódico de seu DNA, e a MMC talvez interfira neste processo em longo prazo. A córnea está constantemente exposta à luz, sendo provável dano ao DNA induzido por radiação ultravioleta.

Jain et al., avaliaram as diferenças quanto à região corneana de aplicação da $M M C^{(8)}$. O estudo comparou a aplicação de MMC per-operatória na forma de discos de $5 \mathrm{~mm}$ de papel filtro ou anéis de $5 \mathrm{~mm}$ de papel filtro com área aberta central de $3 \mathrm{~mm}$. O experimento foi realizado em 24 coelhos e a aplicação da droga foi feita após desepitelização da córnea porém antes da ablação estromal de $100 \mu \mathrm{m}$. Encontraram menor incidência de "haze" no grupo tratado com MMC em padrão anelar na avaliação de duas semanas de pós-operatório. O achado não se manteve nas avaliações de oito e 13 meses de seguimento. $\mathrm{O}$ experimento baseou-se em evidências de que a repopulação de ceratócitos no estroma anterior após trauma corneano ocorre por migração centrípeta de ceratócitos periféricos anteriores ${ }^{(30)}$; a utilização de papel filtro anelar com MMC resultaria em inibição da ativação de ceratócitos circundando o eixo visual, reduzindo sua migração e subsequiente depósito de colágeno.

Em 2001, Xu et al., realizaram PRK para correção de 10 dioptrias miópicas em ambos os olhos de $20 \operatorname{coelhos}^{(9)}$. MMC $0,02 \%$ por período de cinco minutos foi aplicada após ablação com laser apenas no olho direito de cada animal. Avaliação clínica e histopatológica foram realizadas. Menor incidência de "haze" foi observada no grupo que fez uso de MMC ( $<<0,01$ ). Porém, redução importante na população de ceratócitos no estroma anterior foi observada neste grupo até um mês após o procedimento $(\mathrm{p}<0,01)$. Não houve diferença estatística nos dois grupos quanto ao número de ceratócitos entre a $12^{\underline{\underline{a}}}$ e a $26^{\mathrm{o}}$ semana pós-operatória ${ }^{(9)}$.

Outro estudo experimental em coelhos avaliou os efeitos da aplicação única de MMC em duas diferentes concentrações (MMC 0,01\% ou MMC 0,02\%) durante dois minutos após desepitelização corneana mecânica. Encontraram edema endotelial significativo, aumento de pleomorfismo e polimegatismo e redução da contagem endotelial de forma dose dependente durante seguimento de 14 dias $^{(12)}$. Os autores ponderam que o endotélio corneano de coelhos tem capacidade de mitose e que células em divisão são mais sensíveis à ação da MMC; os efeitos em córneas humanas talvez difiram em função da taxa mínima de mitose endotelial.

Em 2003 Kim et al., avaliaram a indução de apoptose de ceratócitos de coelhos em cultura pela $\mathrm{MMC}^{(28)}$. O estudo evidenciou citotoxicidade da MMC estatisticamente significativa nas concentrações de $0,005 \%, 0,01 \%, 0,02 \%, 0,04 \%$ e $0,06 \%$ após 24 horas de exposição aos ceratócitos. MMC $0,01 \%$ apresentou toxicidade crescente diretamente relacionada ao tempo de exposição da droga à córnea. $\mathrm{O}$ experimento evidenciou que a apoptose de ceratócitos dá-se pela via mitocondrial (caspase pathway). Os autores consideram que o processo de apoptose é não inflamatório, fisiológico e consiste na morte de uma célula individual sem dano ao meio adjacente, diferentemente do que ocorre no processo de necrose celular. Acreditam que a MMC previne a mobilização e ativação de ceratócitos remanescentes através da indução de apoptose de ceratócitos ativados. Ponderam ainda que não se sabe se a MMC induz apoptose apenas de ceratócitos em alta atividade proliferativa (ativados), ou se age também sobre ceratócitos inativos; caso atue sobre estes últimos, a redução no número de ceratócitos poderia resultar em afinamentos corneanos anos após uma única aplicação.

Kim et al., também avaliaram os efeitos do uso associado de álcool e MMC sobre os ceratócitos de coelhos em cultu$\mathrm{ra}^{(27)}$. Concluíram que as duas drogas reduzem a viabilidade dos ceratócitos (processo de apoptose) e que a ação das drogas é dependente da concentração e do tempo de exposição das mesmas aos ceratócitos, apresentando efeito sinérgi- 
co. Ainda que estudos "in vitro" utilizando ceratócitos de coelhos tenham limitada aplicação clínica, sugere-se cautela com uso de desepitelização alcoólica (principalmente em concentrações de etanol acima de 30\%) e LASEK em procedimentos fotoablativos em que se faz uso de MMC.

Recentemente, Kim et al., avaliaram os efeitos da MMC no número de ceratócitos e na proliferação de fibroblastos após ceratectomia fotorrefrativa e exposição à radiação ultravioleta $\mathrm{B}$ (UV-B) em coelhos ${ }^{(10)}$. Avaliação imunohistoquímica e microscopia eletrônica foram realizadas. Doze semanas após PRK (-10,0 dioptrias), a aplicação única de MMC $0,02 \%$ por dois minutos reduziu de forma significativa "haze" corneano, número de ceratócitos e fibroblastos, mesmo após exposição UV-B, quando comparado ao grupo que recebeu apenas PRK. A injúria epitelial e o dano cirúrgico induzem apoptose de ceratócitos e a MMC exerce efeito apoptótico adicional sobre o estroma corneano; os efeitos a longo prazo desta depleção são desconhecidos.

\section{Mitomicina C em olhos com cirurgia prévia/uso terapêutico}

Em 2000 foi descrito por Majmudar et al., o uso da MMC para tratamento de fibrose subepitelial recorrente levando à baixa de acuidade visual ${ }^{(13)}$. Os autores relataram o uso de MMC $0,02 \%$ por dois minutos em oito olhos com fibrose subepitelial recorrente (quatro olhos após ceratotomia radial e quatro olhos após PRK), muitos dos quais já haviam sido submetidos a repetidas tentativas de debridamento epitelial, sem sucesso. Após remoção mecânica do epitélio e da fibrose corneana, seguiu-se a aplicação única de MMC. No seguimento entre seis e 25 meses, todos os olhos permaneceram com córnea clara, sem sinais de recorrência da fibrose e com melhora importante da acuidade visual corrigida; nenhum efeito adverso foi relatado.

Em 2002 foi descrito por Marcon, Rapuano, o tratamento de recorrência de distrofia de membrana basal e degeneração nodular de Salzmann com uso de $\mathrm{MMC}^{(14)}$. Os autores relataram um caso de paciente que já havia sido tratado com debridamento epitelial e PTK, apresentando recorrência. Realizouse então novo debridamento epitelial seguido de PTK e MMC $0,02 \%$ durante dois minutos. Após acompanhamento de seis meses, a córnea permaneceu clara sem sinais de recorrência e com melhora da acuidade visual corrigida. Miller et al., também descreveram caso de tratamento e prevenção de recorrência de distrofia de Reis-Bucklers com PTK e viscoelástico seguido de MMC $0,02 \%$ por dois minutos ${ }^{(21)}$. Um ano após o procedimento, o paciente apresentava córnea clara, sem sinais de recidiva da distrofia e com melhora importante da acuidade visual. Jun et al., utilizaram PTK e MMC na interface de LASIK para tratamento de depósitos granulares corneanos exacerbados após LASIK em paciente heterozigótico para o gene da distrofia de Avellino, com bom resultado ${ }^{(31)}$.

Em 2003 Porges et al., usaram aplicação única de MMC em oito pacientes com "haze" corneano intenso após PRK para graus elevados de miopia ${ }^{(15)}$. Todos os pacientes apresentavam "haze" corneano após pelo menos um ano de PRK para alta miopia, baixa de acuidade visual intensa e já haviam sido submetidos a pelo menos um procedimento terapêutico (PRK ou PTK) para controle de "haze". Após remoção mecânica do epitélio corneano, seguiu-se realização de PTK (30 - $40 \mu \mathrm{m}$ de ablação) em $5 \mathrm{~mm}$ corneanos centrais e aplicação de MMC $0,02 \%$ por dois minutos, seguida de copiosa irrigação da superfície ocular. Todos os pacientes apresentaram melhora importante da acuidade visual, redução significativa de "haze" corneano, sem efeitos adversos no seguimento de 12 meses.

Vigo et al., aplicaram MMC em 35 olhos com "haze" importante e regressão após PRK para correção de miopia ${ }^{(16)}$. Vinte destes olhos haviam sido tratados inicialmente com LASIK para correção miópica, permanecendo com grau residual, e então haviam sido submetidos a PRK, desenvolvendo "haze" e regressão. A técnica cirúrgica para tratamento do "haze" consistiu em debridamento epitelial com álcool 20\%, seguido de raspagem superficial para remoção de tecido fibrótico e aplicação de MMC 0,02\% durante dois minutos. Não houve nova ablação com laser. No seguimento de 12 meses, todos os olhos apresentaram melhora importante do "haze", da regressão miópica e da acuidade visual corrigida, sem descrição de efeitos adversos. Os autores comentam que esta concentração e tempo de exposição não parecem induzir nenhuma complicação séria, porém ponderam que a MMC pode acarretar efeitos adversos muitos anos após aplicação.

A MMC também vem sendo utilizada para tratamento de "haze" e regressão após "laser-assisted subepithelial keratomileusis" (LASEK). Mirza et al., descreveram caso de paciente apresentando "haze" que obscurecia detalhes da íris e que foi submetido a debridamento corneano manual e MMC 0,02\% durante dois minutos ${ }^{(32)}$. Apresentou melhora parcial de acuidade visual e "haze". Cinco meses após debridamento corneano e uso de MMC, realizou-se novo debridamento seguido de PTK para remoção de "haze" residual e regularização de superfície corneana, com nova aplicação de MMC. O paciente evoluiu com acuidade visual corrigida de 6/7,5. Os autores utilizam MMC $0,02 \%$ durante dois minutos como profilaxia ao "haze" e regressão em todos os pacientes submetidos a LASEK para correção miópica acima de 6 dioptrias ${ }^{(32)}$.

Alguns autores recentemente descreveram o uso de MMC associado a PRK para tratamento de pacientes após complicações com "flap" de LASIK. Weisenthal et al., publicaram os resultados de série de três pacientes submetidos a PRK e aplicação de MMC 0,02\% por dois minutos duas a 12 semanas após "buttonhole" e "flap" irregular durante confecção de lamela em LASIK $^{(17)}$. O equivalente esférico pré-operatório era de $-5,25$ a $-8,00$ dioptrias. Os pacientes apresentaram acuidade visual sem correção entre 6/6 e 6/7,5 e apenas traços de "haze" no seguimento entre quatro e dez meses. Lane et al., também relataram um caso de PRK e uso de MMC para prevenir "haze", fibrose e regressão em um paciente com "buttonhole" prévio(18). Doze meses após a tentativa de LASIK, com refração (-2,50 esférico), acuidade visual e topografia estáveis, o paciente foi submetido a PRK e uso de MMC 0,02\% durante dois minutos. A desepitelização corneana foi realizada por ablação superficial de $45 \mu \mathrm{m}$. No seguimento de 12 meses, o paciente apresentava acuidade visual sem correção de 6/6, sem evidência de "haze" corneano ${ }^{(18)}$.

Chalita et al., descreveram caso de paciente com "buttonhole" pequeno durante confecção de lamela de LASIK e que recebera aplicação de laser ${ }^{(19)}$. Paciente evoluiu com perda de 
acuidade visual corrigida e queixa de diplopia monocular. Foi submetido a PRK personalizado seguido da aplicação de MMC $0,02 \%$ durante dois minutos, três meses após cirurgia inicial. Um mês após o procedimento, apresentava acuidade visual corrigida de 6/4,5, assintomático, com importante redução das aberrações oculares de alta ordem. Os autores sugerem que PRK personalizado com uso de MMC pode ser uma boa opção após "buttonhole" em que há comprometimento do eixo visual, perda de linhas na acuidade visual corrigida e sintomas visuais. Não houve descrição de toxicidade ou efeitos adversos da MMC.

Solomon et al., também relataram PRK com MMC em paciente após complicações na confecção de lamela de LASIK ("buttonhole") que havia sido submetido a ceratoplastia penetrante dois anos antes por ceratocone ${ }^{(20)}$. Seis meses após o LASIK incompleto, a refração e a acuidade visual estavam estáveis e semelhantes aos valores pré-operatórios (-2,00 DE -3,75 DC @ $\left.45^{\circ}, 6 / 7,5\right)$. O paciente foi submetido a desepitelização alcoólica, ablação com laser e aplicação de MMC $0,02 \%$ durante um minuto. Três meses após o procedimento, o paciente apresentava córnea clara, com acuidade visual de 6/12, acuidade visual corrigida de $6 / 6$ e refração $+0,50 \mathrm{DE}-1,50 \mathrm{DC} @ 42^{\circ}$. Os autores comentam que o desenvolvimento de "haze" prejudica a efetividade do PRK após "buttonhole", especialmente em pacientes submetidos previamente a ceratoplastia penetrante. Sugerem que PRK e uso de MMC no período peroperatório é uma alternativa para pacientes com "buttonhole" após LASIK e transplante de córnea prévio. Os autores ponderam que os efeitos da MMC sobre o endotélio corneano ainda são desconhecidos.

Os resultados de PTK/PRK e uso de MMC após complicações com "flap" de LASIK foram também avaliados por Muller et $\mathrm{al}^{(33)}$. Dez olhos de dez pacientes entraram no estudo. Os pacientes que apresentavam superfície irregular foram tratados com PTK seguido de PRK e MMC 0,02\% por dois minutos. Pacientes sem irregularidade de superfície receberam apenas PRK e aplicação de MMC 0,02\% por dois minutos. Acuidade visual sem correção pré-operatória era de 6/12 a 6/240 e com correção de $6 / 6$ a $6 / 15$. O equivalente esférico pré-operatório era de $+4,00$ a -10,75 dioptrias. Após dez meses de seguimento mínimo, os pacientes apresentaram acuidade visual sem correção entre 6/6 e 6/12 e com correção entre 6/6 e 6/7,5. Não houve relatos de atraso de epitelização corneana, "haze" ou outros sinais de toxicidade.

Acredita-se que nesses casos com finalidade terapêutica, a ação deletéria da MMC sobre as células do estroma anterior resulte em apoptose de muitos miofibroblastos, minimizando a opacidade corneana. Além disso, a ação antimetabólica da MMC impedirá que novos miofibroblastos venham a se formar ${ }^{(34)}$.

Em 2004 Pfister descreveu um caso de edema corneano permanente após PTK e uso de colírio de MMC 0,02\% por seis dias após o procedimento ${ }^{(22)}$. O paciente foi descrito como portador de distrofia microcística de Cogan em olho esquerdo. Apresentava ainda fuso de Krukenberg bilateral, com pressão intraocular normal em ambos os olhos. Foi submetido inicialmente a PTK (ablação estromal de $20 \mu \mathrm{m}$ ) em olho esquerdo, com desenvolvimento de hipertensão ocular córtico-dependente e "haze" importante. O paciente foi então submetido a novo tratamento a laser, seguido do uso de colírio de MMC 0,02\% iniciado dois dias após o procedimento. A posologia de uso foi uma gota quatro vezes ao dia nos primeiros dois dias, intervalo de um dia sem uso, seguido de mais três dias de uso duas vezes ao dia. Defeito epitelial corneano estava presente durante o período de uso da MMC. Avaliação oftalmológica dois meses após o tratamento evidenciou edema corneano epitelial e estromal difuso. Contagem endotelial era de 700 células por $\mathrm{mm}^{2} \mathrm{em}$ olho esquerdo e 2.200 células por $\mathrm{mm}^{2} \mathrm{em}$ olho direito.

Não há relatos na literatura de doença corneana (descompensação endotelial, ectasia, defeito epitelial persistente, deficiência límbica) induzida pela MMC quando usada em aplicação corneana única no momento cirúrgico com finalidade óptica/ refrativa. Este é o primeiro caso descrito de descompensação corneana após uso de MMC para fins refrativos; porém o paciente fez uso contínuo de MMC colírio durante período pósoperatório (na presença de defeito epitelial), há dúvidas quanto ao diagnóstico da patologia de base do paciente e quanto ao uso ou não de MMC também no ato cirúrgico. São questionáveis ainda a concentração da MMC usada (manipulada), a aderência do paciente à prescrição e a contagem endotelial préoperatória (não há microscopia especular prévia). De qualquer forma, a inocuidade da MMC na córnea e especificamente em relação ao endotélio corneano precisa ser melhor estudada.

Recentemente, descreveu-se o primeiro caso de PRK associado a MMC para correção de ametropia resisual pós-transplante penetrante de córnea em ceratocone ${ }^{(35)}$. Paciente apresentava refração de +7,00 DE-4,75 DC @ $125^{\circ}$ dez meses após ceratoplastia penetrante, sem suturas corneanas. Foi submetido a PRK seguido da aplicação de MMC 0,02\% com bom resultado ${ }^{(35)}$.

\section{Mitomicina C em olhos sem cirurgia prévia/uso profilático}

Em 2002, Carones et al., publicaram o primeiro estudo de uso per-operatório profilático de MMC após PRK para inibir a formação de "haze" corneano em olhos com miopia moderada a alta ${ }^{(23)}$. Sessenta olhos sem tratamento prévio com equivalente esférico entre -6,00 e -10,00 dioptrias foram aleatoriamente distribuídos para receber apenas PRK (30 pacientes) ou PRK seguido da aplicação intra-operatória de MMC 0,02\% durante dois minutos ( 30 pacientes). Paquimetria corneana pré-operatória maior que $480 \mu \mathrm{m}$ e cálculo corneano residual após o procedimento de pelo menos $400 \mu \mathrm{m}$ eram critérios de inclusão. Olhos que receberam MMC foram intencionalmente hipocorrigidos em 10\%. "Excimer laser" LadarVision (Alcon laboratórios) foi utilizado em todos os casos. Não houve diferença significativa quanto ao tempo para reepitelização, dor, efeitos adversos, ou toxicidade entre os dois grupos. Houve diferença estatisticamente significativa em relação ao equivalente esférico $(\mathrm{p}=0,05)$, acuidade visual sem correção (seis meses após procedimento, $60 \%$ dos pacientes tratados com MMC e 30\% dos pacientes do grupo controle apresentavam acuidade visual de $6 / 6, \mathrm{p}=0,001)$, acuidade visual corrigida $(\mathrm{p}=0,0006)$ e presença de "haze" $(\mathrm{p}=0,01)$, com melhores resultados no grupo que recebeu aplicação de MMC. Os autores sugerem que o PRK associado ao uso profilático de MMC pode ser uma alternativa para pacientes que apresentam graus 
miópicos moderados a altos e córneas cuja espessura não permite a realização segura de LASIK com zona óptica adequada $^{(23)}$. Sugerem também que o procedimento pode ser interessante para cirurgias personalizadas, em que uma menor interferência cicatricial é desejável. Os autores ponderam ainda que o tempo de seguimento do estudo foi curto (seis meses) e recomendam o uso cuidadoso de MMC enquanto a segurança da técnica cirúrgica não for atestada a longo prazo.

Em 2004 foi realizada por Camellin, a análise comparativa dos resultados de LASEK e LASEK com MMC em pacientes míopes $^{(24)}$. Oitenta e seis olhos foram submetidos a LASEK seguido de "pincelada" de MMC 0,01\% após ablação corneana para correção miópica média de $-6,80 \pm 3,00$ dioptrias. Cem olhos com grau miópico estatisticamente semelhante foram submetidos apenas a LASEK. "Excimer laser" Nidek EC-5000 (Nidek) foi utilizado em todos os casos. Para avaliação dos resultados, cada grupo foi subdividido em olhos com miopia baixa (-1,20 a $-10,80 \mathrm{D}$, grau I) e miopia alta (-5,60 a $-15,50 \mathrm{D}$, grau II). Com um ano de seguimento, olhos grau I em refração dinâmica apresentaram equivalente esférico médio de $+0,50 \pm 0,93$ dioptrias no grupo da $\mathrm{MMC}$ e de $0 \pm 0,34$ dioptrias no grupo controle (hipercorreção estatisticamente significativa, $\mathrm{p}<0,05$ ). Olhos grau II não apresentaram diferença entre os grupos. Menor incidência de "haze" foi observada nos olhos que receberam aplicação de MMC $(p<0,05)$. Não houve diferença em relação à dor e à reepitelização corneana nos dois grupos. Análise das aberrações de alta ordem pelo topógrafo Keratron Scout (Optikon 2000) em diâmetro pupilar de $5 \mathrm{~mm}$ evidenciou aumento significativo das aberrações de alta ordem no grupo da MMC $(\mathrm{p}<0,05 \%)$. O autor comenta que a MMC induz hipercorreção, sugerindo redução de $20 \%$ na programação cirúrgica quando do uso da droga. Questiona ainda se a MMC não teria toxicidade corneana crônica, lembrando que o seguimento após uso do antimetabólito em cirurgia refrativa é pequeno. Este estudo, porém, deve ser avaliado com cautela, pois a subdivisão em olhos com miopia baixa e alta para análise dos resultados é pouco clara, o autor não estabelece em que grupo se enquadrariam olhos entre $-5,60$ e $-10,80$ dioptrias.

Em 2004 Hashemi et al., descreveu o uso profilático de MMC associado ao PRK para redução de "haze" em alta miopia ${ }^{(25)}$. Cinqüenta e quatro olhos com equivalente esférico entre $-5,00$ e $-9,88$ D foram submetidos a PRK ("excimer laser" Technolas $217-\mathrm{C})$ e uso de MMC $0,02 \%$ durante dois minutos. Nenhum dos olhos apresentava espessura corneana para realização de LASIK com leito estromal residual de $250 \mu \mathrm{m}$ (paquimetria central préoperatória média de $488 \pm 11,9 \mu \mathrm{m}$ ). Todos os olhos foram programados para 5\% de hipocorreção. Seis dias após procedimento, todos os olhos apresentaram epitelização corneana completa. No seguimento de seis meses, 39 olhos ( $81,3 \%$ ) estavam entre $\pm 0,5 \mathrm{D}$ da emetropia e 45 olhos $(93,7 \%)$ estavam entre $\pm 1,00 \mathrm{D}$ da emetropia. Acuidade visual sem correção de $6 / 6$ ou melhor foi observada em 37 olhos $(77,1 \%)$ e 48 olhos (100\% dos pacientes que retornaram seis meses após procedimento) apresentaram 6/12 ou melhor. Não houve perda de linhas de visão corrigida. Apenas dois olhos apresentaram traços de "haze" um mês após cirurgia, que desapareceu nos retornos seguintes. Sensibilidade ao contraste nas frequiências espaciais de 6 e 12 ciclos por grau melhorou aos seis meses de cirurgia em relação aos valores préoperatórios. Não houve relatos de complicações. Os autores orientam cautela quanto ao uso de MMC enquanto resultados de longo prazo não estiverem disponíveis e concluem que PRK com MMC é uma alternativa para pacientes com alta miopia e córneas que não permitam realização segura de LASIK.

Estudo recente de Gambato et al., avaliou o impacto funcional e morfológico do uso de MMC após PRK para tratamento de olhos alto míopes ${ }^{(26)}$. Entraram no estudo 72 olhos de 36 pacientes com alta miopia bilateral (entre - 7,00 e - 14,25 D), aleatoriamente distribuídos para PRK com MMC no per-operatório (MMC $0,02 \%$ por dois minutos) em um olho e apenas PRK no olho contralateral. O estudo foi duplo-cego durante todo o seguimento (média de 18 meses). Olhos tratados com MMC não receberam corticosteróides durante período pós-operatório; olhos tratados apenas com PRK receberam fluormetolona $2 \%$ colírio três vezes ao dia no primeiro mês, duas vezes ao dia durante segundo mês e uma vez ao dia até completar três meses do procedimento. $\mathrm{O}$ estudo não demonstrou diferença entre tempo de reepitelização corneana (até cinco dias), dor ou efeitos adversos entre os dois grupos. Acuidade visual sem correção foi melhor no grupo que fez uso de MMC ( $\mathrm{p}=0,03)$ até o último seguimento. Incidência e quantificação de "haze" foi maior no grupo que recebeu apenas PRK $(\mathrm{p}=0,02)$ um ano após procedimento. A análise por microscopia confocal (ConfoScan 3,0) evidenciou maior refletividade estromal anterior (aumento da densidade local e proliferação de ceratócitos ativados, com núcleo e corpo celular de alta reflectividade) no grupo que recebeu apenas PRK quando comparado ao grupo que recebeu também MMC no seguimento de 12 e 24 meses. Além disso, o grupo que recebeu também MMC apresentou retorno mais rápido e de forma mais estável aos níveis pré-operatórios. Avaliação epitelial e endotelial por microscopia confocal com 24 e 36 meses da cirurgia não demonstraram alteração morfológica. Quanto à sensibilidade ao contraste, os dois grupos apresentaram perda significativa um mês após a cirurgia; o grupo tratado com MMC apresentou recuperação mais rápida, não diferindo significativamente dos valores pré-operatórios três meses após o procedimento. Os autores concluem que a aplicação única de MMC $0,02 \%$ reduz de forma segura a formação de "haze" em olhos alto míopes no seguimento de 36 meses; porém ponderam que efeitos degenerativos tardios podem ocorrer anos após uso de MMC, semelhante aos efeitos radioativos.

Acredita-se que em casos de uso profilático a MMC bloqueie a replicação dos fibroblastos, impedindo sua transformação em miofibroblastos. É preciso cautela com uso da droga, pois o bloqueio da replicação de fibroblastos a longo prazo pode resultar em defeituosa repopulação celular e conseqüente diminuição da celularidade estromal, levando à possível alteração estrutural do tecido corneano ${ }^{(34)}$. É provável que em casos profiláticos menor concentração e tempo de exposição corneana da droga sejam suficientes, uma vez que apenas efeito citostático sobre os ceratócitos do estroma anterior é desejável. 


\section{CONCLUSÕES}

A aplicação única de mitomicina $\mathrm{C}$ durante cirurgia fotoablativa de superfície tem se mostrado uma opção segura e eficiente para fins terapêuticos em olhos com opacidade corneana préexistente e/ou profiláticos em olhos com alto risco de desenvolvimento de opacificação corneana pós-operatória. Novos estudos ainda são necessários para determinar a menor concentração e o menor tempo de exposição corneana eficaz da droga para cada fim óptico/refrativo. $\mathrm{O}$ uso da mitomicina $\mathrm{C}$ em cirurgia fotoablativa de superfície corneana deve ser cauteloso até que seguimento de longo prazo avalie sua inocuidade tardia.

\section{ABSTRACT}

Mitomycin C is an antimetabolite agent that blocks DNA and RNA replication and protein synthesis. It has been used in several ophthalmologic areas, and recently as a modulator of corneal wound healing in excimer laser surgeries. A single application of mitomycin $\mathrm{C}$ during surface corneal photoablative surgery seems a safe and efficient therapeutic option for eyes with corneal opacity and/or as prophylaxis in eyes with high risk for corneal opacity development. The use of this drug in photoablative surgery should be cautious until long-term safety results have been reported. The present text presents a review about corneal wound healing with the use of mitomycin $\mathrm{C}$.

Keywords: Cornea; Wound healing; Mitomycin/therapeutic use; Refractive errors/surgery; Excimer laser photorefractive surgery/methods

\section{REFERÊNCIAS}

1. Calabresi P, Chabner BA. Antineoplastic agents. In: Goodman Gilman A, Rall TW, Nies AS, Taylor P, editors. Pharmacological basis of therapeutics. $8^{\text {th }}$ ed. New York: Pergamon Press; 1990. p.1247-8.

2. Bindlish R, Condon GP, Schlosser JD, D'Antonio J, Lauer KB, Lehrer R. Efficacy and safety of mitomycin-C in primary trabeculectomy: five-year follow-up. Ophthalmology. 2002;109(7):1336-41; discussion 1341-2.

3. Akura J, Kaneda S, Matsuura K, Setogawa A, Takeda K, Honda S. Measures for preventing recurrence after pterygium surgery. Cornea. 2001;20(7):703-7.

4. Shields CL, Naseripour M, Shields JÁ. Topical mitomycin C for extensive, recurrent conjunctival-corneal equamous cell carcinoma. Am J Ophthalmol 2002;133(5):601-6

5. Donnenfeld ED, Perry HD, Wallerstein A, Caronia RM, Kanellopoulos AJ, Sforza PD, et al. Subconjunctival mitomycin $\mathrm{C}$ for the treatment of ocular cicatricial pemphigoid. Ophthalmology. 1999;106(1):72-8.

6. Schipper I, Suppelt C, Gebbers JO. Mitomycin C reduces scar formation after excimer laser $(193 \mathrm{~nm})$ photorefractive keratectomy in rabbits. Eye. 1997;11 (Pt 5):649-55.

7. Sadeghi HM, Seitz B, Hayashi S, LaBree L, McDonnell P. In vitro effects of mitomycin-C on human keratocytes. J Refract Surg. 1998;14(5):534-40.

8. Jain S, McCally RL, Connolly PJ, Azar DT. Mitomycin C reduces corneal light scattering after excimer keratectomy. Cornea. 2001;20(1):45-9.

9. Xu H, Liu S, Xia X, Huang P, Wang P, Wu X. Mitomycin C reduces haze formation in rabbits after excimer laser photorefractive keratectomy. J Refract Surg. 2001;17(3):342-9.

10. Kim T, Pak JH, Lee SY, Tchab H. Mitomycin C - induced reduction of keratocytes and fibroblasts after photorefractive keratectomy. Invest Ophthalmol Vis Sci. 2004;45(9):2978-84.

11. Talamo JH, Gollamudi S, Green WR, Cruz ZDL, Filatov V, Stark WJ. Modulation of corneal wound healing after excimer laser keratomileusis using topical mitomycin C and steroids. Arch Ophthalmol. 1991;109(8):1141-6.

12. Chang SW. Early corneal edema following topical application of mitomycinC. J Cataract Refract Surg. 2004;30(8):1742-50.

13. Majmudar PA, Forstot L, Dennis RF, Nirankari VS, Damiano RE, Brenart R et al. Topical mitomycin-C for subepithelial fibrosis after refractive corneal surgery. Ophthalmology. 2000;107(1):89-94.

14. Marcon AS, Rapuano CJ. Excimer laser phototherapeutic keratectomy retreatment of anterior basement membrane dystrophy and Salzmann's nodular degeneration with topical mitomycin C. Cornea. 2002;21(8):828-30.

15. Porges Y, Bem-Haim O, Hirsh A, Levinger S. Phototherapeutic keratectomy with mitomycin $\mathrm{C}$ for corneal haze following photorefractive keratectomy for myopia. J Refract Surg. 2003;19(1):40-3.

16. Vigo L, Scandola E, Carones F. Scraping and mitomycin $\mathrm{C}$ to treat haze and regression after photorefractive keratectomy for myopia. J Refract Surg. 2003;19(4):449-54.

17. Weisenthal RW, Salz J, Sugar A, Mandelberg A, Furlong M, Bagan S, et al Photorefractive keratectomy for treatment of flap complications in laser in situ keratomileusis. Cornea. 2003;22(5):399-404.

18. Lane HA, Swale JA, Majmudar PA. Prophylactic use of mitomycin-C in the management of a buttonholed LASIK flap. J Cataract Refract Surg. 2003; 29(2):390-2.

19. Chalita MR, Roth AS, Krueger RR. Wavefront-guided surface ablation with prophylactic use of mitomycin $\mathrm{C}$ after a buttonhole laser in situ keratomileusis flap. J Refract Surg. 2004;20(2):176-81.

20. Solomon R, Donnenfeld ED, Perry HD. Photorefractive keratectomy with mitomycin $\mathrm{C}$ for the management of a LASIK flap complication following a penetrating keratoplasty. Cornea. 2004;23(4):403-5.

21. Miller A, Solomon R, Bloom A, Palmer C, Perry HD, Donnenfeld ED. Prevention of recurrent Reis-Bucklers dystrophy following excimer laser phototherapeutic keratectomy with topical mitomycin C. Cornea. 2004;23(7):732-5

22. Pfister RR. Permanent corneal edema resulting from the treatment of PTK corneal haze with mitomycin. Cornea. 2004;23(7):744-7.

23. Carones F, Vigo L, Scandola E, Vacchini L. Evaluation of the prophylactic use of mitomycin $\mathrm{C}$ to inhibit haze formation after photorefractive keratectomy. J Cataract Refract Surg. 2002;28(12):2088-95.

24. Camellin M. Laser epithelial keratomileusis with mitomycin C: indications and limits. J Refract Surg. 2004;20(5 Suppl):S693-8.

25. Hashemi H, Taheri SMR, Fotouhi A, Kheiltash A. Evaluation of the prophylactic use of mitomycin-C to inhibit haze formation after photorefractive keratectomy in high myopia: a prospective clinical study. BMC Ophthalmol. 2004;4(1):12-6.

26. Gambato C, Ghirlando A, Moretto E, Busato F, Midena E. Mitomycin C modulation of corneal wound healing after photorefractive keratectomy in highly myopic eyes. Ophthalmology. 2005;112(2):208-18.

27. Kim TI, Tchah H, Cho EH, Kook MS. Evaluation for safety of cultured corneal fibroblasts with cotreatment of alcohol and mitomycin C. Invest Ophthalmol Vis Sci. 2004:45(1):86-92.

28. Kim TI, Tchah H, Lee AS, Sung K, Cho BJ, Kook MS. Apoptosis in keratocytes caused by mitomycin C. Invest Ophthalmol Vis Sci. 2003; 44(5):1912-7.

29. McDermott ML, Wang J, Shin DH. Mitomycin and the human corneal endothelium. Arch Ophthalmol. 1994;112(4):533-7.

30. Kratz-Owens KL Hageman GS, Schanzlin DJ. An in-vivo technique for monitoring keratocyte migration following lamellar keratoplasty. Refract Corneal Surg. 1992;8(3):230-4.

31. Jun RM, Tchah H, Kim TI, Stulting RD, Jung SE, Seo KY et al. Avellino corneal dystrophy after LASIK. Ophthalmology. 2004;111(3):463-8.

32. Mirza MA, Qazi MA, Pepose JS. Treatment of dense subepithelial corneal haze after laser-assisted subepithelial keratectomy. J Cataract Refract Surg. 2004;30(3):709-14.

33. Muller LT, Candal EM, Epstein RJ, Dennis RF, Majmudar PA. Transepithelial phototherapeutic keratectomy/photorefractive keratectomy with adjunctive mitomycin-C for complicated LASIK flaps. J Cataract Refract Surg. 2005;31(2):291-6

34. Netto MV, Junior RA, Chalita MR, Krueger RR, Wilson SE. Resposta cicatricial corneana em diferentes modalidades de cirurgia refrativa. Arq Bras Oftalmol. 2005;68(1):140-9.

35. Solomon R, Donnenfeld ED, Thimons J, Stein J, Perry HD. Hyperopic photorefractive keratectomy with adjunctive topical mitomycin $\mathrm{C}$ for refractive error after penetrating keratoplasty for keratoconus. Eye Contact Lens. 2004;30(3):156-8 